\title{
LESÕES EXTENSAS DO MANGUITO ROTADOR: AVALIAÇÃO DOS RESULTADOS DO REPARO ARTROSCÓPICO
}

\author{
EXTENSIVE ROTATOR CUFF INJURIES: AN EVALUATION OF \\ ARTHROSCOPIC REPAIR OUTCOMES
}

Alberto Naoki Miyazaki', Marcelo Fregoneze², Pedro Doneux Santos ${ }^{3}$, Luciana Andrade Silva ${ }^{3}$, Eduardo, Cesar Moreira Mariz Pinto Rodrigo Tormin Ortiz ${ }^{4}$, Sergio Luiz Checchia ${ }^{5}$

\section{RESUMO}

Objetivos: Avaliar o resultado do tratamento cirúrgico das lesões extensas do manguito rotador por via de acesso artroscópica. Métodos: Entre junho de 1998 e outubro de 2006 foram reavaliados 61 pacientes com lesões extensas do manguito rotador submetidos a tratamento cirúrgico por técnica artroscópica pelo Grupo de Ombro e Cotovelo do Departamento de Ortopedia e Traumatologia da Faculdade de Ciências Médicas da Santa Casa de Misericórdia de São Paulo. Foram incluídos no estudo todos os pacientes com acometimento de pelo menos dois tendões ou com retração de pelo menos dois tendões até a borda da cavidade glenoidal e com no mínimo 12 meses de seguimento. Resultados: Pelos critérios de avaliação da UCLA, 54 (89\%) pacientes tiveram resultados excelentes e bons; nenhum, regular; e sete (11\%), ruins. Verificou-se índice de satisfação de 92\% dos pacientes. A mobilidade articular no período pós-operatório dos pacientes passou da média de elevação de $93^{\circ}$ para $141^{\circ}$, a média da rotação lateral foi de $32^{\circ}$ para $48^{\circ}$ e a média da rotação medial, de L1 para T10. Essas diferenças foram estatisticamente significantes. Conclusão: O reparo artroscópico das lesões extensas do manguito rotador traz resultados satisfatórios na maioria dos pacientes, com alto grau de satisfação.

Descritores - Manguito rotador; Artroscopia/método; Estudos de avaliação

\section{ABSTRACT}

Objectives: To assess the outcomes of the surgical treatment of extensive rotator cuff injuries through arthroscopy. Methods: Between June 1998 and October 2006, 61 patients with extensive rotator cuff injuries and submitted to surgical arthroscopy technique by the Shoulder and Elbow Group of the Department of Orthopaedics and Traumatology, Santa Casa de Misericórdia Medical School were reassessed. The study included all patients with at least two tendons affected or with retraction at least on two tendons up to the glenoidal cavity edge and with at least 12 months of follow-up. Results: According to UCLA's evaluation criteria, 54 (89\%) patients showed excellent or good outcomes; no fair outcome in none of the patients; and seven (11\%) poor outcomes. A satisfaction rate of $92 \%$ was reported. Postoperative joint motion went from a mean lifting value of $93^{\circ}$ to $141^{\circ}$, the mean lateral rotation went from $32^{\circ}$ to $48^{\circ}$ and the mean medial rotation went from L1 to T10. These differences were regarded as statistically significant. Conclusion: The arthroscopic repair of extensive rotator cuff injuries leads to satisfactory outcomes for most of the patients, with a high satisfaction degree.

Keywords - Rotator cuff; Arthroscopy/ method; Evaluation studies

1 - Professor Doutor Assistente do Departamento de Ortopedia e Traumatologia da Faculdade de Ciências Médicas da Santa Casa de São Paulo; Chefe do Grupo de Cirurgia de Ombro e Cotovelo.

2 - Professor Assistente do Departamento de Ortopedia e Traumatologia da Faculdade de Ciências Médicas da Santa Casa de São Paulo; Assistente do Grupo de Cirurgia de Ombro e Cotovelo.

3 - Médico Ortopedista; Assistente do Grupo de Cirurgia de Ombro e Cotovelo do Departamento de Ortopedia e Traumatologia da Faculdade de Ciências Médicas da Santa Casa de São Paulo.

4 - Médico Estagiário do Grupo de Cirurgia de Ombro e Cotovelo do Departamento de Ortopedia e Traumatologia da Faculdade de Ciências Médicas da Santa Casa de São Paulo.

5 - Professor Doutor Adjunto e Chefe de Clínica do Departamento de Ortopedia e Traumatologia da Faculdade de Ciências Médicas da Santa Casa de São Paulo; Consultor Acadêmico do Grupo de Cirurgia de Ombro e Cotovelo.

Trabalho realizado no Departamento de Ortopedia e Traumatologia da Faculdade de Ciências Médicas da Santa Casa de São Paulo, "Pavilhão Fernandinho Simonsen. Correspondência: Santa Casa de Misericórdia de São Paulo, Departamento de Ortopedia e Traumatologia, "Pavilhão Fernandinho Simonsen”,

Rua Dr. Cesário Mota Júnior, 112 - 01220-020 - São Paulo, SP. E-mail: ombro@ombro.med.br 


\section{INTRODUÇÃO}

O termo “lesões extensas do manguito rotador" (LEMR) tem sido utilizado largamente para identificar lesões grandes cuja reparação é particularmente difícil, portanto, com prognóstico incerto. Hawkins et al definem como extensas as lesões superiores a cinco centímetros de comprimento $^{(1)}$. Devido à variação de tamanho dos pacientes e de técnicas de mensuração, Gerber et al acreditam ser mais apropriada a definição do tamanho em termos da quantidade de tendões envolvidos na lesão; classificam como extensas as que envolvem no mínimo dois tendões ${ }^{(2)}$.

As LEMR evoluem, especialmente nos pacientes jovens, com grande incapacidade funcional, com perda de força de elevação e dor crônica; geralmente, o tratamento clínico não leva a bons resultados ${ }^{(3)}$.

A utilização da via aberta no reparo das lesões do manguito rotador pode levar a complicações amplamente discutidas na literatura, tais como: dano ao músculo deltoide, artrofibrose e dor residual ${ }^{(4)}$, de difícil correção no período pós-operatório. A técnica híbrida, com acromioplastia pela via artroscópica e sutura da lesão por miniincisão, é outra opção de tratamento, porém, não é isenta de complicações ${ }^{(5,6)}$. A artroscopia tem-se sedimentado como opção para tratamento dessas lesões por combinar máxima visualização da articulação com mínimo dano tecidual $^{(4)}$. Pacientes submetidos a procedimentos artroscópicos para sutura de lesões do manguito rotador têm menos dor no período pós-operatório e retorno mais rápido da função e das atividades da vida diária ${ }^{(7,8)}$.

O reparo das LEMR pode ser, tecnicamente, desafiador devido às retrações dos cotos tendinosos, fibrose bursal, atrofia muscular e degeneração gordurosa da musculatura $^{(9)}$. Devido a essas características, as lesões extensas, eventualmente, são chamadas de "irreparáveis”, o que nem sempre corresponde à realidade.

As opções de tratamento para essas lesões extensas do manguito rotador são: conservador, desbridamento articular com tenotomia do tendão da cabeça longa do músculo bíceps braquial, reconstrução com enxerto homólogo, transferências de músculos da cintura escapular, tentativa de reparo parcial ou até mesmo completo do manguito rotador, sempre que possível, e artroplastia reversa do ombro em casos de exceção ${ }^{(10-14)}$. Quando técnicas apropriadas de mobilização são empregadas, a grande maioria dessas lesões pode ser reparada completa ou parcialmente ${ }^{(9,11,15,16)}$.

O objetivo deste trabalho é avaliar os resultados do tratamento cirúrgico artroscópico das “lesões extensas” do manguito rotador.

\section{MÉTODOS}

No período de junho de 1998 a outubro de 2006, 71 pacientes com lesões extensas do manguito rotador foram submetidos a tratamento cirúrgico artroscópico; desse total, 61 foram reavaliados pelo Grupo de Ombro e Cotovelo do Departamento de Ortopedia e Traumatologia da Faculdade de Ciências Médicas da Santa Casa de São Paulo - "Pavilhão Fernandinho Simonsen”. Foram considerados critérios de inclusão neste estudo pacientes com lesões do manguito rotador com comprometimento de mais de dois tendões ou que tivessem retração dos cotos tendinosos de pelo menos dois deles ao nível da borda superior da cavidade glenoidal, identificada através da imagem de ressonância magnética realizada no período pré-operatório ou durante o procedimento artroscópico. Foram considerados critérios de exclusão pacientes submetidos a tratamento cirúrgico artroscópico com lesões que acometessem apenas um tendão ou que a retração desses tendões não chegasse ao nível da glenoide, ou aqueles que tivessem menos de 12 meses de seguimento pós-operatório (Tabela 1).

Entre os pacientes, 27 eram do sexo masculino (44\%) e 34, do feminino (56\%). A média de idade foi de 64 anos e quatro meses, variando de 45 a 82 anos. O tempo de dor no período pré-operatório variou de uma semana a 192 meses, com média de 30 meses e 10 dias. O lado dominante foi acometido em 51 (84\%) casos. Entre as lesões, 42 (69\%) foram decorrentes de trauma ou esforço físico no ombro. Dos pacientes acometidos, 25 (41\%) praticavam esportes que utilizam o membro superior.

A mobilidade articular pré-operatória foi avaliada pelos parâmetros descritos por Hawkins e Bokos ${ }^{(17)}$. A elevação variou de $0^{\circ}$ a $160^{\circ} \mathrm{com}$ média de $92^{\circ}$; a rotação lateral, de $0^{\circ}$ a $90^{\circ}$, com média de $32^{\circ}$; e a rotação medial avaliada conforme o nível vertebral alcançado pelo paciente com o polegar, variou do trocânter maior ipsilateral a T7, com média de L1. Nem todos os pacientes foram submetidos a exame de ressonância magnética no período pré-operatório, especialmente os primeiros da série.

Todos os pacientes foram submetidos ao procedimento cirúrgico em posição de "cadeira de praia”, sob anestesia geral associada a bloqueio do plexo braquial. Todos os pacientes foram submetidos à inspeção intraarticular, em que foram visualizadas as lesões associadas. Nos casos em que foi observada lesão do tendão da cabeça longa do bíceps, foi realizada a tenotomia, seguida de tenodese, antes da reparação do manguito. Após a inspeção intra-articular, foi feita a inspeção subacromial, sendo as lesões classificadas de acordo com 
os critérios de inclusão. Após desbridamento das partes moles, mobilização dos tendões e preparo do leito ósseo no tubérculo maior, a sutura foi feita com o uso de âncoras, pontos tendão-tendão ou combinações dessas técnicas. A tenodese do tendão da cabeça longa do bíceps, acromioplastia e ressecção da extremidade distal da clavícula foram consideradas procedimentos associados, conforme a necessidade (Figuras 1 e 2).

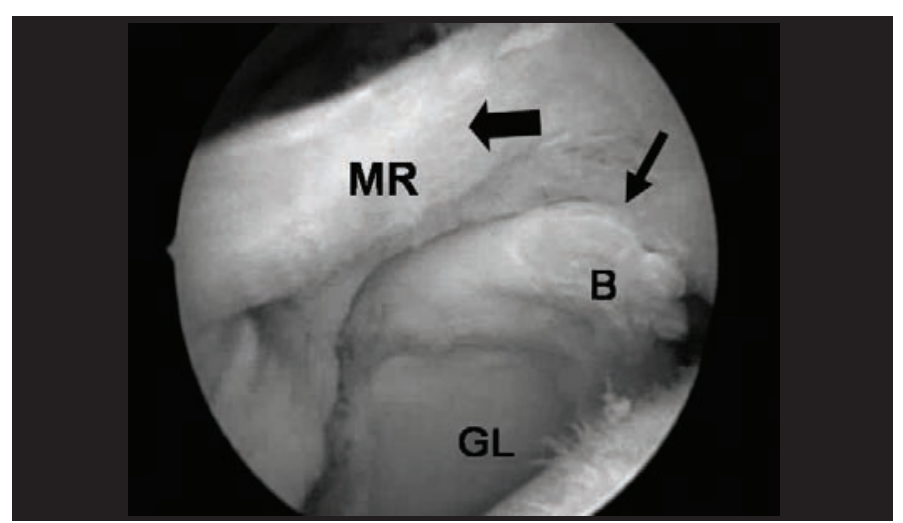

Figura 1 - Imagem intra-operatória de cirurgia por via artroscópica para reparo de lesão extensa do manguito rotador (MR) com visão através do portal lateral: seta larga- borda da lesão do MR na altura da borda da cavidade glenoidal (GL); seta fina- origem do tendão da cabeça longa do bíceps (B) submetido à tenotomia.

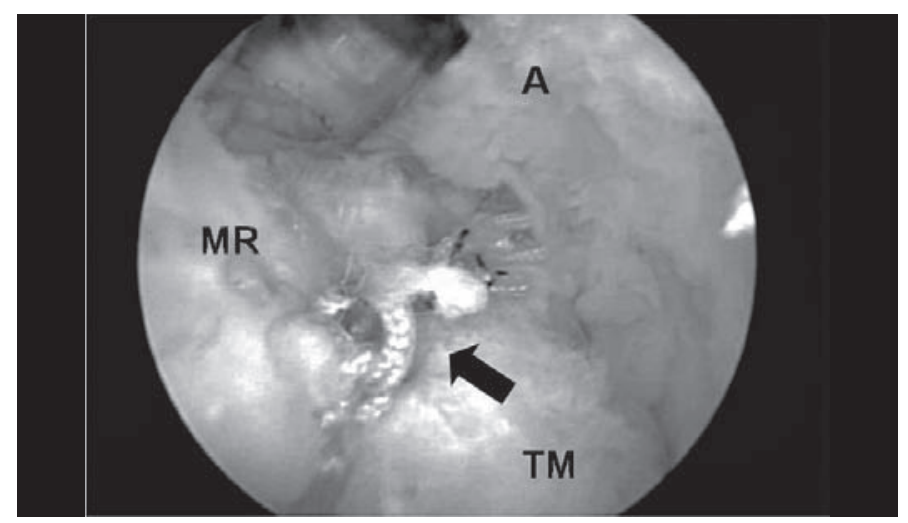

Figura 2 - Imagem intra-operatória da cirurgia para reparo de lesão extensa do manguito rotador (MR) no tubérculo maior (TM) por via artroscópica pelo portal posterior monstrando os pontos realizados na sutura da lesão (seta). Reparo realizado com 3 âncoras e 6 pontos. ( $\mathrm{A}=$ ACRÔMIO).

Dos pacientes, 39 (64\%) possuíam lesões que comprometiam total ou parcialmente a inserção do tendão do músculo subescapular. A acromioplastia foi realizada em 52 (85\%) pacientes e a ressecção da extremidade distal da clavícula, em 18 (29\%) destes. A tenodese do tendão da cabeça longa do músculo bíceps braquial foi realizada em 20 (32\%) pacientes. Em 13 (21\%) a tenodese foi realizada na sutura da lesão do manguito rotador e, em sete (11\%), com o uso de parafuso de interferência de biotenodese (Arthrex ${ }^{\circledR}$ ). Em oito (13\%) pacientes foi realizada somente a tenotomia do tendão da cabeça longa do músculo bíceps braquial, pois este já se encontrava aderido, provavelmente, no sulco intertubercular; em outros oito pacientes (13\%), esse tendão estava ausente durante a inspeção articular.

Em todos os pacientes a sutura da lesão foi realizada por meio de âncoras; o número utilizado variou de um a cinco, com média de três. O número de pontos realizados variou de quatro a 12, com média de sete. Em 50 casos foram realizados também pontos tendão-tendão para aproximar as bordas da lesão antes da sutura ao osso, com média de dois pontos tendão-tendão, variando de um a quatro pontos. Em dois casos não foi possível realizar a sutura completa do tendão ao osso, sendo então utilizado o princípio da “ponte pênsil” para o reparo parcial ${ }^{(11)}$.

O tempo médio de imobilização no período pósoperatório, com tipoia funcional, foi de sete semanas, variando entre quatro e 12 semanas.

No período pós-operatório os pacientes foram reavaliados clinicamente pelo método da University of California at Los Angeles (UCLA) e a mobilidade articular, pelos parâmetros descritos por Hawkins e Bokos ${ }^{(17)}$.

Na análise estatística, foi utilizado o teste dos postos sinalizados de Wilcoxon para verificar possíveis diferenças entre dois momentos de observação, para as variáveis de interesse, e a análise de correlação de Spearman para persquisar o nível de relacionamento entre os pares de variáveis formados. Adotamos o nível de significância de $5 \%(0,05)$ para a aplicação dos testes estatísticos.

\section{RESULTADOS}

Quando avaliamos os resultados dos 61 pacientes operados, obtivemos pontuação média (UCLA) de 31,2 (seis a 35 pontos). Foram considerados excelentes em $67 \%$ dos casos e bons em 22\%. Em 11\% dos casos, com UCLA entre seis a 20, o resultado foi considerado como mau e nenhum caso, como regular (Figuras 3 e 4).

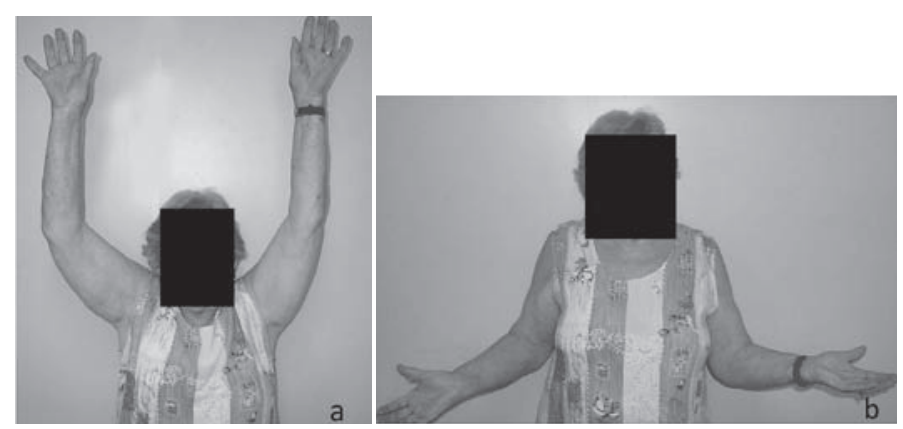

Figura 3 - Paciente (caso 60) no período pós-operatório 23 meses de reparo de lesão extensa do manguito rotador do ombro direito, com bom resultado: a- elevação ativa; b- rotação lateral ativa. 

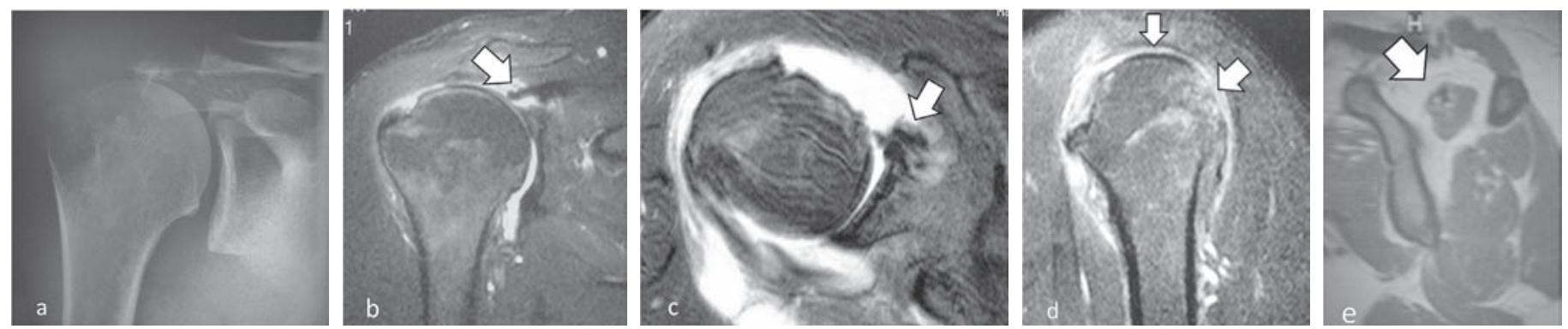

Figura 4 - Exames de imagem do período pré-operatório de paciente (caso 60) com lesão extensa do manguito rotador (MR): aradiografia ântero-posterior mostra a ascensão da cabeça do úmero; b-ressonância magnética (RNM) em corte coronal T2, mostra retração do tendão do supra-espinal até a borda da cavidade glenoidal (seta) e ascensão da cabeça do úmero; c- RNM axial T2 , lesão do tendão subescapular (seta); d- RNM sagital T2, lesão do MR incluindo os tendões do supra-espinal e infra-espinal (setas); RNM sagital T1, degeneração gordurosa maior do que $50 \%$ do músculo supra-espinal (seta).

A amplitude média de movimento, na avaliação pósoperatória, foi de $141^{\circ}$ de elevação, variando de $45^{\circ}$ a $160^{\circ}$, rotação lateral de $48^{\circ}$, de $0^{\circ}$ a $80^{\circ}$, e rotação medial de T10, de L5 a T5. O seguimento médio foi em torno de 36 semanas, variando de 12 a 108 meses.

A idade, presença de trauma ou esforço e acromioplastia associada não evidenciaram correlação estatisticamente significante com o resultado. Os pacientes com lesão do subescapular obtiveram ganho maior na rotação medial (um nível) em relação aos com subescapular íntegro, mas essa diferença foi estatisticamente não significante.

Complicações foram observadas em nove casos (15\%), tais como: deiscência da sutura tendinosa, evidenciada em cinco casos; artropatia do manguito rotador, em dois casos; e capsulite adesiva, em outros dois.

\section{DISCUSSÃO}

O tratamento das lesões extensas é controverso, principalmente em relação à técnica a ser utilizada. As opções de tratamento são as mais variadas, desde não cirúrgico, desbridamento aberto associado à descompressão subacromial, desbridamento artroscópico associado ou não a descompressão subacromial, reparo parcial do manguito rotador, reparo aberto, reparo pela técnica da mini-incisão, reparo artroscópico, tenotomia do bíceps e técnicas combinadas ${ }^{(7,10-13,15,18,19,20)}$, além de mais recentemente podermos utilizar a prótese reversa ${ }^{(14)}$.

O tamanho das LEMR não é o único fator a ser considerado na escolha do método de tratamento; a mobilidade e a qualidade dos tendões e dos ventres musculares correspondentes são fatores importantes para avaliar a possibilidade de reparação dessas lesões ${ }^{(3)}$.

Bigliani et $\mathrm{al}^{(9)}$ obtiveram $85 \%$ de bons e excelentes resultados no tratamento cirúrgico aberto das lesões extensas do manguito rotador e salientaram a importância da integridade do músculo deltoide para o programa de reabilitação. Em contrapartida, Bennett ${ }^{(10)}$ observou taxa de 95\% de resultados satisfatórios com o tratamento artroscópico das LEMR em um estudo prospectivo com dois a quatro anos de seguimento, não encontrando diferença estatisticamente significativa entre lesões que envolviam ou não o tendão subescapular.

Em 2001, Burkhart ${ }^{(18)}$ ressalta a vantagem da visualização da lesão por via artroscópica e o reconhecimento de seu formato para o emprego das técnicas de fechamento, geralmente por convergência de margens associada ou não com o reparo tendão-osso, atingindo $92 \%$ de bons e excelentes resultados. Da mesma forma, Jones e Savoie $^{(12)}$, em um estudo prospectivo com 60 pacientes, obtiveram $88 \%$ de bons e excelentes resultados, com índice de satisfação de $98 \%$ dos casos.

Mais recentemente, outros autores publicaram trabalhos utilizando a técnica da liberação artroscópica do intervalo dos rotadores para melhor mobilização dos tecidos retraídos e verificaram resultados satisfatórios em estudos preliminares ${ }^{(9,21)}$.

Nossos resultados foram excelentes ou bons na grande maioria dos casos, atingindo em torno de 89\% (54 ombros), valor semelhante quando comparado com nossos resultados em trabalhos anteriores com operação aberta e mini-incisão. Em relação à movimentação ativa, ocorreu aumento médio de $52^{\circ}$ na elevação anterior e de $16^{\circ}$ na rotação lateral. Quanto à rotação medial, houve incremento médio de quatro níveis vertebrais na amplitude articular. $\mathrm{O}$ acréscimo nessas três direções da amplitude de movimento mostrou-se estatisticamente significativo.

Em dois pacientes não foi possível realizar o fechamento de toda a lesão e foram tratados com o reparo parcial preconizado por Burkhart ${ }^{(11)}$ e, mesmo assim, obtiveram resultados satisfatórios (casos 47 e 55). Outro paciente (caso 3) apresentou deiscência da sutura com três anos de seguimento pós-operatório, após episódio traumático de luxação anterior do ombro, mas optou por não reoperar e evoluiu favoravelmente com UCLA 34. 
Verificamos maus resultados em sete pacientes (11\%). Dois desses (casos 24 e 43) evoluíram com deiscência da sutura. Um paciente (caso 27) não seguiu as orientações médicas, retirando a imobilização antes do tempo recomendado. Outra paciente (caso 44), portadora de hepatite C e com perfil psicológico depressivo, evoluiu com capsulite adesiva e, mesmo após o tratamento com bloqueio anestésico seriado do nervo supraescapular, persiste com resultado ruim. Evidentemente, e de acordo com Boileau et $\mathrm{al}^{(22)}$, sabemos que o índice de deiscência das suturas do manguito rotador, por via aberta ou artroscópica, é maior do que o por nós evidenciado; isso se deve, fundamentalmente, por não realizarmos imagens de ressonância magnética pós-operatória em todos, seja por impossibilidade econômica, seja pela não aceitação do paciente em realizar o exame. Apenas aqueles pacientes que evoluíram insatisfatoriamente foram submetidos a tal exame (Figura 5).

Além dos quatro pacientes citados anteriormente, observamos mais dois resultados insatisfatórios nos casos 6 e 37, os quais evoluíram com artropatia pós-lesão do manguito rotador. Em ambos foi indicada artroplastia reversa, mas apenas em um destes, foi realizada.

Obtivemos o alívio da dor em 87\% dos casos, com índice de satisfação de aproximadamente 92\%, evidenciando a efetividade do tratamento cirúrgico artroscópico para as lesões extensas do manguito rotador.

Quanto à imobilização pós-operatória, em geral deixamos o paciente com uma tipoia funcional (em abdução e rotação neutra do ombro) por cerca de seis a oito semanas, fazendo exercícios passivos para o bíceps braquial. Um paciente retirou a tipoia com apenas quatro

\section{REFERÊNCIAS}

1. Hawkins RJ, Misamore GW, Hobeika PE. Surgery for full thickness rotator-cuff tears. J Bone Joint Surg Am. 1985;67(9):1349-55.

2. Gerber C, Fuchs B, Hodler J. The results of repair of massive tears of rotator cuff. J Bone Joint Surg Am. 2000;82(4):505-15.

3. Checchia SL, Doneux Santos P, Miyazaki NA, Fregoneze M, Silva LA, Mussi Filho S, Faria AC, Ribeiro Junior GFF. Tratamento cirúrgico das lesões extensas do manguito rotador pela via de acesso deltopeitoral. Rev Bras Ortop. 2003;38(5):252-260.

4. Checchia SL, Doneux Santos P, Miyazaki AN, Fregoneze M, Silva LA, Ishi M, Braga SR, Nascimento LG. Avaliação dos resultados obtidos na reparação artroscópica das lesões do manguito rotador. Rev Bras Ortop. 2005;40(5):229-38.

5. Miyazaki AN, Doneux Santos P, Saito RY Kussakawa D, Checchia SL. Acromioplastia por via artroscópica e reparo das lesões do manguito rotador por "miniincisão". Rev Bras Ortop.1999;34(7):415-20.

6. Bittar ES. Arthroscopic management of massive rotator cuff tears. Arthroscopy. 2002;18(9 Suppl 2):104-6.

7. Galatz LM, Ball CM, Teefey SA, Middleton WD, Yamaguchi K. The outcome and repair integrity of completely arthroscopically repaired large and massive rotator cuff tears. J Bone Joint Surg Am. 2004;86(2):219-24.

8. Rokito AS, Cuomo F, Gallagher MA, Zuckerman JD. Long-term functional outcome of repair of large and massive chronic tears of the rotator cuff. J Bone Joint Surg Am. 1999;81(7):991-7.

9. Bigliani LU, Cordasco FA, Mcilveen SJ, Musso ES. Operative treatment of failed repair of massive rotator cuff. J Bone Joint Surg Am. 1992;74(10):1505-15.

10. Bennett WF. Arthroscopic repair of massive rotator cuff tears: A prospective cohort with 2- and 4- year follow-up. Arthroscopy. 2003;19(4):380-90.

11. Burkhart SS. Arthroscopic treatment of massive rotator cuff tears. Clin Orthop Relat Res. 2001;(390):107-18

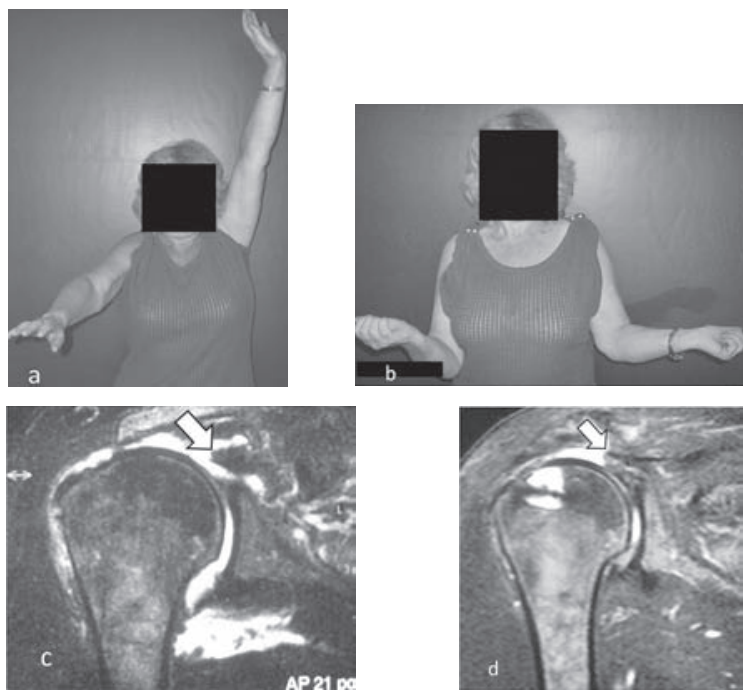

Figura 5 - Paciente (caso 43) no período pós-operatório (PO) de 57 meses de reparo por via artroscópica de lesão extensa do manguito rotador com má evolução: a- elevação ativa; b- rotação lateral ativa; c- ressonância magnética (RNM) pré-operatória: observa-se a retração da lesão até borda da cavidade glenoidal (seta); d- RNM PO: re-ruptura do manguito rotador (seta).

semanas e acabou tendo resultado cirúrgico muito ruim. Outro caso foi o da paciente que evoluiu com capsulite adesiva e, devido a dor, não conseguiu retirar a tipoia e permaneceu imobilizada por 12 semanas.

\section{CONCLUSÃO}

O tratamento artroscópico das lesões extensas do manguito rotador leva a $89 \%$ de excelentes e bons resultados e proporciona aumento da amplitude do movimento do ombro.

12. Jones CK, Savoie FH 3rd. Arthroscopic repair of large and massive rotator cuff tears. Arthroscopy. 2003;19(6):564-71.

13. Tauro JC. Arthroscopic repair of large rotator cuff tears using the interval slide technique. Arthroscopy. 2004;20(1):13-21.

14. Wall B, Nové-Josserand L, O`Connor DP, Edwards TB, Walch G. Reverse total shoulder arthroplasty: a review of results according to etiology. J Bone Joint Surg Am. 2007;89(7):1476-85.

15. Lo IK, Burkhart SS. Arthroscopic repair of massive, contracted, immobile rotator cuff tears using single and double interval slides: technique and preliminary results. Arthroscopy. 2004;20(1):22-33.

16. Burkhart SS, Athanasiou KA, Wirth MA. Margin convergence: A method of reducing strain in massive rotator cuff tears. Arthroscopy. 1996;12(3):335-8.

17. Hawkins RJ, Bokos DJ. Clinical evaluation of shoulder problems. In: Rockwood CA Jr, Matsen FA 3rd. The shoulder 2nd ed. Philadelphia: Saunders; 1998. p.175-80.

18. Burkhart SS. Partial repair of massive rotator cuff tears: The evolution of a concept. Orthop Clin North Am. 1997;28(1):125-32.

19. Snyder SJ, Murray TF, Lajtai G, Mileski RM. Arthroscopic repair of medium to large full-thickness rotator cuff tears: Outcome at 2- to 6-year follow up. J Shoulder Elbow Surg. 2002;11(1):19-24.

20. Boileau P, Brassart N, Watkinson DJ, Carles M, Hatzidakis AM, Krishnan SG. Arthroscopic repair of full-thickness tears of the supraspinatus: Does the tendon really heal? J Bone Joint Surg. Am. 2005;87(6):1229-40.

21. Walch G, Edwards TB, Boulahia A, Nove-Josserand L, Neyton L, Szabo I. Arthroscopic tenotomy of the long head of the biceps in the treatment of rotator cuff tears: Clinical and radiographic results of 307 cases. J Shoulder Elbow Surg. 2005; 14(3):238-46.

22. Boileau P, Baque F, Valerio L, Ahrens P, Chuinard C, Trojani C. Isolated arthroscopic bíceps tenotomy or tenodesis improves symptoms in patients with massive irreparable rotator cuff tears. J Bone Joint Surg Am. 2007;89(4):747-57. 